\title{
RADIO OBSERVATIONS OF MASSIVE OB STARS
}

\author{
IAN D. HOWARTH \\ Dept. Physics \& Astronomy \\ University College London \\ Gower Street \\ London WC1E 6BT \\ England
}

\author{
ALEXANDER BROWN \\ JILA \\ University of Colorado \\ Boulder \\ Colorado 80309-0440 \\ $U S A$
}

ABSTRACT. The mass-loss rates of $O$ stars and $B$ supergiants are of interest because of their influence on the evolution of these massive stars (among other matters). In principle, the 'safest: (i.e. most model-independent) method of determining $M$ is to measure the free-free emission from stellar winds at radio wavelengths. This method is complicated, however, by the existence of poorly understood non-thermal emission in some stars, and by the possibility of hydrogen recombination in the winds of $B$ supergiants.

We are in the process of carrying out a VLA survey of OB stars, initially at $3.5 \mathrm{~cm}$, to a flux limit of $\sim 0.1 \mathrm{mJy}$. Because all our targets should have thermal emission at detectable levels (based on mass-loss rates from Howarth \& Prinja 1989 and terminal velocities from Prinja. Barlow \& Howarth 1990), the survey is yielding an unbiassed estimate of the frequency of non-thermal emission. The improved sensitivity of our survey over earlier work defines the $\log \dot{M}-\log L$ relationship much more precisely than was previously possible, over a large range in luminosities; and allows us to make definitive statements on recombination in B supergiant winds. Our sample includes the first radio detections of an OC star, of a massive X-ray binary, and of thermal emission from a main-sequence star.

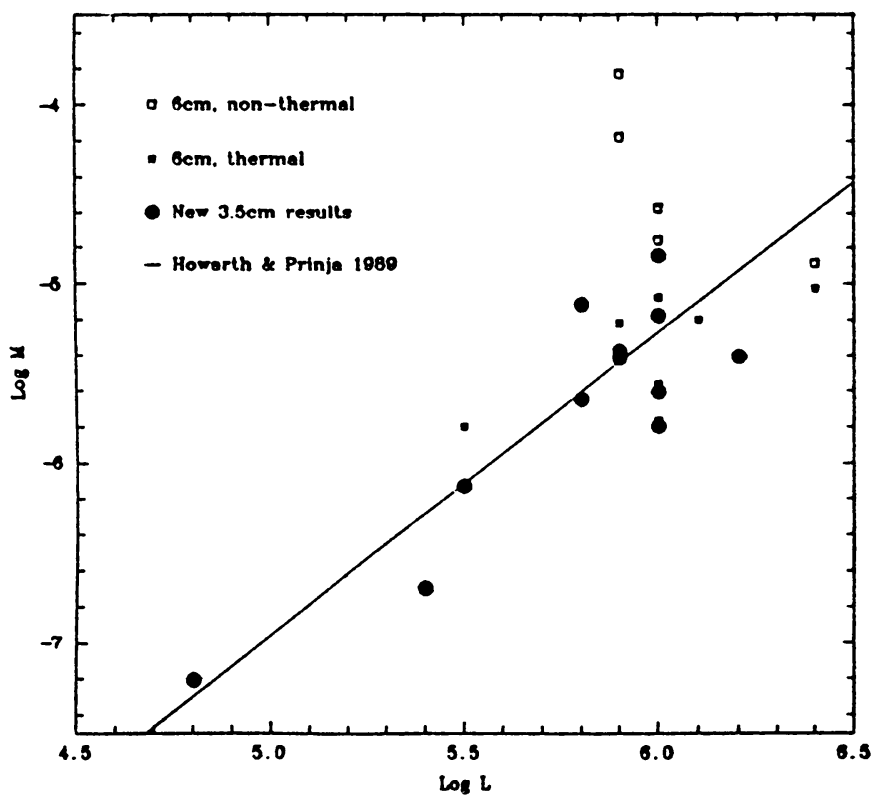

315

K. A. van der Hucht and B. Hidayat (eds.),

Wolf-Rayet Stars and Interrelations with Other Massive Stars in Galaxies, 315.

(C) 1991 IAU. Printed in the Netherlands. 